\title{
Development of a Paratransit Microsimulation Patron Accessibility Analysis Tool for Small and Medium Sized Communities
}

\author{
Jeffrey J. LaMondia \\ The University of Texas at Austin \\ Dept of Civil, Architectural and Environmental Engineering \\ 1 University Station C1761 \\ Austin, TX 78712-0278 \\ Phone: 512-471-4535, Fax: 512-475-8744 \\ E-mail: jlamondia@mail.utexas.edu \\ and \\ Chandra R. Bhat* \\ The University of Texas at Austin \\ Dept of Civil, Architectural and Environmental Engineering \\ 1 University Station C1761 \\ Austin, TX 78712-0278 \\ Phone: 512-471-4535, Fax: 512-475-8744 \\ E-mail: bhat@mail.utexas.edu \\ *corresponding author
}

July 2009

Revised November 2009 


\begin{abstract}
Paratransit is a critical form of transportation for mobility-impaired, low income, and small/ medium sized communities. Paratransit systems face many challenges that restrict how well they can serve their community, including limited funding, aging fleets, limited to no level of service standard assessments, and few practical modeling/planning practices. This paper discusses a transferable paratransit microsimulation patron accessibility analysis tool designed to address these challenges. The tool calculates paratransit patron accessibility (defined as paratransit patrons' perceived ease of access to reach desired activities and destinations) by simulating and measuring daily paratransit patron travel patterns based on service fleet and region information. The tool further allows providers to evaluate patron accessibility for any combination of population groups, travel purposes, and times of day. Transit providers can use the tool to determine how well paratransit patrons are served and the most efficient ways to improve service. The microsimulation framework, including the system of simulation models, the supporting data, and application to Brownsville, Texas are described in detail.
\end{abstract}




\section{INTRODUCTION}

Paratransit, also known as demand-responsive transit or dial-a-ride, is a critical form of transportation for mobility-impaired, low-income, elderly, and rural populations. This type of service, which transports riders through an on-demand basis, is commonly employed in four main markets: 1) for the general public in rural areas that are not dense enough to support a fixed route transit system, 2) for the general public in urban areas acting independently of a fixed route transit system, 3) for the general public in urban areas as a feeder to a fixed route transit system, and 4) as ADA complementary services required by the 1991 Americans with Disabilities Act (ADA) (1).

The way paratransit typically operates is as follows: Patrons of the paratransit service call their transit operator, usually at least 24 hours ahead of time, to schedule their trip. As trips are scheduled, transit operators use optimization software to update the paratransit vehicles' routes for the given day. Paratransit vehicle drivers receive this manifest at the beginning of each day, which informs them where they need to go. Schedules can sometimes even become repetitive "subscription type services" in areas where paratransit has been long established due to numerous repeat patrons.

There are many variations of paratransit service, depending on the needs of the area. Some operators provide point-to-point service, transporting patrons to and from specific points similar to a taxi (2). Others provide route-deviation service, picking up and dropping off patrons at specific locations but always returning to a loosely defined route (much like a bus). Service can be further customized by choosing to pick up and drop off patrons at the requested origins/destination, at convenient locations (including a fixed-route bus stop), or any combination of these (1).

Paratransit service is quite prevalent in the US. For instance, there were over 86.6 million unlinked paratransit trips in 2005 (3). Yim and Khattak further reported that over 370,000 vehicles and 22,884 private paratransit operators served these patrons (4). Regardless of the markets and locations served by these paratransit operators, all of them face similar challenges: they contend with limited funding, understaffing, aging fleets, a lack of technical support, limited to no level of service standard assessments, and few practical modeling/planning practices. These challenges are especially present in small and medium sized communities, where the largest percentages of residents rely on paratransit service. In fact, over $21 \%$ of the United States' population currently resides in small and medium sized communities (5), and these numbers are projected to increase as such areas continue to develop as nationally critical economic centers (6). As populations within such regions grow, the challenges to paratransit operators will be amplified, potentially resulting in reduced mobility and stunted economic growth in paratransit service areas. Therefore, it is critically important that small and region communities take a proactive approach to transit planning.

Much of the previous work within demand response transit research focuses on paratransit operations, including fleet distribution, scheduling, and other supply-side factors. Studies of how to reduce costs and optimize routing began in the 1970s, when computer-aided scheduling software was first introduced, and have continued ever since (7). Many studies of paratransit best practices exclusively consider operational improvements (8-10). Models of customer serviceability (11), vehicle serviceability (12), and optimal fleet size (13) have been developed around these operational characteristics as well.

This emphasis on operational improvements is partly due to the limited budgets and staffing of transit operators in small and medium sized communities (2). But, it is also due to the 
fact that national funding and performance reviews are heavily based on these operational measures. However, paratransit operations provide only half of the picture. Paratransit operators and community planners must also consider patron travel needs to comprehensively assess the effectiveness of their transit program. In particular, just because a paratransit system is costeffective does not automatically mean that all patrons are getting their preferred service. Fortunately, a few researchers and operators are taking cues from fixed-route transit research and beginning to recognize the importance of considering patron-level performance (i.e. accessibility) measures when assessing paratransit systems (14-16). Many simple patron-level performance accessibility measures exist, including number of on-time pickups, excessive travel times, arrival delays, and unmet demand $(17,18)$. However, these measures tend to be aggregated across the entire service region and simply entail benchmarking (i.e. recording and comparing values) over time. In fact, much of the literature that includes patron-level accessibility measures are primarily concerned with identifying the most inexpensive, efficient, and effective methods for recording this information (16-19). Small and medium sized communities are most likely to collect these measures by conducting phone interviews with patrons, collecting comment cards, or performing occasional surveys (2).

More importantly, these simple benchmark accessibility measures fail to consider accessibility from patrons' perspectives. While travel times and on-time pickups are important, it is the paratransit patrons' perceptions of these accessibility characteristics that ultimately affect their behavior. As a result, current accessibility measures cannot be used to predict or anticipate how changes in their service operations, fleet, or region will affect patrons' accessibility. This is especially critical for paratransit operators seeking funding opportunities based on improving paratransit patron accessibility, such as the Rural Transportation Accessibility Incentive Program sponsored by the Federal Transit Administration (FTA) (20), as they need to be able to determine the most effective applications of these funds to improve patron travel satisfaction.

The most robust accessibility measures evaluate the number of travel opportunities to which patrons have access as well as their ability to move between these travel opportunities (19, 21). The challenge in applying this level of patron accessibility to paratransit is the fact that paratransit travel opportunities and characteristics change every day depending on where and when patrons request service, making measuring patron accessibility difficult. Therefore, in order to capture this level of detail, one must simulate actual daily paratransit travel patterns. The simulated travel characteristics may then be used to measure paratransit patron accessibility.

Recently, a number of related advanced methods for calculating paratransit performance measures have emerged. One important topic of analysis in this regard has been the forecasting of rural transit demand based on population characteristics, either through linear regression models, factoring methods, or other means. Most earlier studies on this topic predict the total number of paratransit patrons within a region $(1,13,22,23)$, while some studies predict the number of different types of paratransit patrons within a region, such as the elderly, subsidized, low income, youth, and mobility-impaired $(22,24)$. These earlier forecasting studies provide insight into whether demand is being met and the latent need of the service region. Other studies develop methods for calculating typical travel characteristics for patrons based on distances between trip origins and destinations (13) which can later be compared. Southworth et al. (25) even distinguished differences in travel characteristics (i.e. costs, distance, time, safety, mobility) based on trip purpose and available modes in his cost-benefit analysis tool. Fu (26) developed a complete simulation system for paratransit travel based on dynamic scheduling. However, his 
tool is designed to evaluate the impact that different types of technologies have on scheduling practices.

Still, to the knowledge of the authors, no complete paratransit patron accessibility microsimulation model currently exists. As indicated earlier, paratransit patron accessibility measures are especially important in small and medium sized communities because the land uses in these regions are different from those in urban areas $(27,28)$. For this reason it would be especially important to not only consider paratransit patron travel characteristics but also how these characteristics are related to the service area land use patterns and where paratransit patrons want to travel.

The current research builds upon previous work and presents a paratransit microsimulation analysis tool that measures paratransit patron accessibility (defined as paratransit patrons' perceived ease of access to reach desired activities and destinations). In order to accommodate the changing daily paratransit patrons' travel characteristics, the analysis tool is comprised of a microsimulation framework as well as accessibility measurements. Furthermore, these two components incorporate detailed spatial and individual patron elements to calculate patron accessibility for various types of individuals, times of day, trip purposes, and, most importantly, spatial areas. As a result, operators can determine the quality of paratransit service across the service area and identify the most cost-effective ways to improve their service from a patron-perspective.

Ultimately, the analysis tool packages the microsimulation framework and accessibility measurements together into a user-friendly Microsoft Access database application. This format was primarily selected because it is familiar to many transit operators and allows the tool to be transferable, practical, and valuable for all small and medium communities. The tool runs the microsimulation based on user-entered fleet and service area characteristics, selected timeframe, and defined season. Naturally, analyses over longer timeframes offer more precision simply because there are more iterations to compare, but take more computation time and processing power. The benefit of the stand-alone analysis tool application is that it does not require advanced training or technology, such as GPS or routing software, to implement.

The rest of the paper is structured as follows: Section 2 first discusses the data used to formulate the paratransit microsimulation analysis tool. Sections 3 and 4 detail the tool components: the microsimulation framework for paratransit patron travel and methods for calculating patron accessibility, respectively. Microsimulation patron accessibility is then evaluated in a case study in Section 5. Finally, conclusions and future research are presented in Section 6.

\section{MICROSIMULATION ANALYSIS TOOL FORMULATION}

The underlying models of the paratransit microsimulation patron accessibility analysis tool were developed using a combination of spatial GIS data and actual recorded patron trip data, collected from the paratransit system operating in Brownsville, Texas. Because the analysis tool utilizes both types of data, users are able to evaluate paratransit patron accessibility at the patron-level and zone-based disaggregate scales. Even though the data used in the tool is extremely detailed, the research team selected data sources that would be easy for transit operators and planners to collect or replicate as well as be straightforward for non-technical planners to implement. The steps involved in formatting the spatial GIS and patron trip data are outlined in the following paragraphs. 
Spatial GIS data was first collected from both the US Census Bureau and Brownsville Urban System (BUS), the transit provider within Brownsville, in the form of three main shapefiles for roads, census block groups, and fixed-route transit routes. A number of steps were used to format and clean the shapefiles: First, these shapefiles were formatted and clipped to the area within the paratransit service region seen in Figure 1. Second, sociodemographics data for each census block group were added from the census SF1 demographic library. Third, land uses for each census block group, in the form of zoning, were added. Land uses included manufacturing, commercial, retail, apartments, and general residential. Fourth, distances between every pair of census block group centroids were calculated. Finally, the distance from each census block group centroid to the nearest fixed-route transit line was calculated.

The patron trip data, consisting of a detailed list of patrons as well as a complete log of all completed trips over an 8 month period, was collected from BUS. The travel log contained all 28,751 paratransit patron trips from June 1, 2006 to January 31, 2007. The list of patrons contained every patron ever recorded from 2001 to 2007. Duplicates were removed, and only those who had taken one of the trips during the 8 months of the travel log were selected. As a result, the final list contained 380 unique patrons, with gender, mobility, and home location data.

Finally, the spatial GIS and patron trip data were combined. In this last step, patron home locations and trip origins and destinations were geocoded in ArcGIS. By merging these files, the research team was able to identify exactly where paratransit patrons were living and traveling to. This connection was a critical component in developing the system of models used to measure paratransit patron accessibility, described in the following section.

\section{MICROSIMULATION FRAMEWORK}

This section provides an overview of the framework used to simulate paratransit patrons' travel patterns over a 24-hour period. The microsimulation is comprised of a series of probability models, linear models, and discrete choice models (presented in Figure 1) that build off each other to predict patron characteristics and decisions. These models were estimated using the actual paratransit trip data collected from Brownsville, Texas. By the end of this simulation, the analysis tool generates a table of patrons being served, their demographics, origin and destination zones, trip purpose and time of day, travel characteristics, and whether they are able to be accommodated on this day or not. Of course, the microsimulation requires data consisting of 1) a list of fleet vehicles, including capacity, service schedules, reliability (i.e., how often the vehicle will be out of service for repairs), efficiency (i.e., how many patrons this vehicle can pick up in an hour), hours of operation, handicapped-patron accessibility, daily cost of operation and revenue, and 2) a table of census block groups within the service area, including land uses/ zoning and population demographics, distance to transit, and distance between zones. It is important to recognize that the patron trip data and scheduling records collected from Brownsville Urban System used to develop the tool are not required of other transit agencies to run the tool. The series of seven specific models included in this module are discussed in turn next.

\subsection{Total Patron Demand Generation}

The simulation begins with predicting the total number of patrons requesting a paratransit trip from each census block group based on the population demographics of the area. The explanatory variables in this linear regression model can be classified in three groups: general demographic characteristics, demographic percentages within the specific census block group 
population, which describe the individual character of the block group, and demographic percentages within the entire region population, which describe the block group in reference to all other block groups. This linear regression, presented in Table 1, was estimated from the geocoded BUS patron list and census block group characteristics. The results indicate that census block groups that have larger total populations, older populations, larger average household sizes, and are closer to fixed transit routes produce more paratransit patrons. The impact of renters, married households with children, and different aged populations on patron generation are also significant, but the roles of these population factors vary greatly depending on spatial scale. More specifically, the effect that these population groups have on patron generation varies depending on whether these populations make up a significant percentage of the block group population, a significant percentage of the regional population, or both. For example, block groups that have a high percentage of their population that rents produce fewer paratransit patrons. However, if the block group contains most of the regional population of renters, it will produce more paratransit patrons. These spatial interactions and variations suggest that although a large percentage of paratransit patrons tend to be older and without access to a vehicle, paratransit is also used by patrons of all ages and sociodemographic characteristics. This conclusion is similar to what Koffman et al. (23) found in their regional paratransit demand forecasting model.

\subsection{Gender and Mobility Identification}

The next model identifies whether each patron is either male or female and if he/she is mobile or mobility-impaired. Mobility-impaired patrons have some kind of mobility impairment that requires the use of an aid to get around, such as a cane or wheelchair. Based on the actual BUS patron list, patrons have a $33.04 \%$ probability of being a mobility-impaired female, $25.80 \%$ probability of being a mobile female, $24.06 \%$ probability of being a mobility-impaired male, and a $17.10 \%$ probability of being a mobile male. The simulation stores these probabilities as a continuous number scale from 0.00 to 100.00 , based on the fact that the probabilities sum to $100 \%$. As a result, 0.00 through 33.04 on the gender and mobility number scale is associated with being a mobility-impaired female, 33.05 through 58.84 is associated with being a mobile female, 58.85 through 82.90 is associated with being a mobility-impaired male, and 82.91 through 100.00 is associated with being a mobile male. The simulation then draws a random number between 0.00 and 100.00 for each patron and assigns his/her identity based on where this random number falls on the gender and mobility number scale. For example, if the random number drawn for a patron was 62.30, then that patron would be identified as a mobilityimpaired male.

This technique is also applied to the following models in order to simulate patron's paratransit travel decisions. The decision models first calculate the probability a patron will select each alternative. The simulation then converts these probabilities into a continuous number scale, similar to the one described above, and a random number between 0.00 and 100.00 is drawn. Finally, each patron's decision is determined based on where this random number falls on the probability number scale [this is a standard procedure used in microsimulation methods; see, for example, (29)].

\subsection{Trip Purpose Estimation}

Trip purposes are estimated in the third model of the simulation. In a method similar to the previous model, the simulation assigns trip purposes to patrons based on gender, mobility, and 
probabilities derived from the BUS travel log. The possible trip purposes include church/meeting, education/school, seeking recreation, medical/therapy, shopping, and work. Interestingly, most trips taken using paratransit, regardless of the traveler's gender and mobility, are for medical/ therapy. Mobile patrons use paratransit to travel for work considerably more than mobility-impaired patrons. Mobility-impaired patrons, on the other hand tend to use paratransit for recreation-related travel more than mobile patrons (we are not presenting the detailed statistics of trip purposes because of TRB word limit considerations; interested readers can obtain this information from the authors).

\subsection{Destination Zone Assignment}

The simulation continues with a model of where each paratransit patron is most likely to travel. This model was estimated from the BUS paratransit travel logs and geocoded origin and destination locations. In this multinomial logit discrete choice model, presented in Table 2, each census block group is treated as a destination alternative. As such, the model is applied to calculate a patron's probability of choosing each census block group. The probabilities of traveling to each possible destination are then used to assign each patron's destination. The results in Table 2 indicate that both male and mobility-impaired patrons prefer destinations that are less populated and closer to their origin, relative to female and mobile patrons, respectively. These results suggest that not only do men and mobility-impaired patrons have less patience for traveling on transit, but that most of their trips are task-oriented, such as going to work or a medical appointment; they spend less time traveling to places to simply visit friends and family. Zoning land uses, categorized as residential, apartments, commercial (including public buildings and space), retail businesses, and manufacturing (including industrial), are important factors in this model as well. Patrons traveling for different purposes are more likely to go to areas zoned to support that type of activity. For example, patrons traveling for recreation purposes are more likely heading to census block groups where outdoor spaces, school sports fields, and gyms are located, i.e. more commercial, retail, and apartment zoning. Similarly, patrons traveling to shop are considerably more likely to visit census block groups with high percentages of land area zoned for retail business. The full results of the model can be found in Table 2. Interestingly, distances were not significant in this model, meaning that paratransit patrons make destination decisions regardless of the distance they might have to travel to get there. This modeling process repeats for each individual patron until all patrons are assigned a destination census block group.

\subsection{Time of Day Allocation}

Patrons' choice of when to travel is simulated next using another multinomial logit discrete choice model. This model determines when each patron is most likely to travel, based on his/her demographics, trip purpose, and destination. The choices for this model are a) to travel out and return in the morning, b) to travel out in the morning and return in the afternoon, or c) to travel out and return in the afternoon. The probabilities of choosing to travel during these three times are calculated for each patron using the discrete choice model specification listed in Table 3. The probabilities calculated for each time of day are then used to assign his/her travel time of day. Patrons are more likely to complete their travel during the morning if they are mobile, traveling long distances, and if they are traveling for task-oriented activities, such as work, education, or medical purposes. On the other hand, patrons are more likely to complete their travel during the afternoons if they are traveling longer distances, are mobility-impaired, and if they are traveling for leisure-oriented activities, such as church/meeting, recreation, or shopping 
purposes. Mobility-impaired patrons are also likely to spread their travel across the entire day. However, this becomes more likely if they are traveling shorter distances but are pursuing longer activities, such as a church/meeting or recreation. This modeling process repeats for each individual patron until all patrons are assigned a time of day for travel. This discrete choice model was estimated using the BUS paratransit travel log.

\subsection{Patron Travel Scheduling}

Once patrons' demographics, trip purposes, and destination and time of day choices are completed, this model loads the patrons into the paratransit vehicles by first randomly selecting patrons from the list to simulate a calling order. The maximum number of patrons able to be served during the specified timeframes is calculated from fleet size, vehicle capacities, probability a vehicle is out-of-service, number of patrons each vehicle can serve per hour, and paratransit hours of operation. Mobility-impaired patrons are only able to be scheduled onto vehicles identified as being handicapped-accessible. When a patron is randomly selected, the analysis tool schedules them only if their travel time of day and mobility needs can be met; otherwise the tool will continue to select a new patron until either vehicle capacities are met or all unscheduled patrons' travel needs cannot be met. The model assumes that each trip consists of two legs: one from the home to the destination and the second from the destination to the home. This assumption is consistent with most paratransit trips. In fact, close to $90 \%$ of the trips logged by BUS were only 2 legs.

\subsection{Travel Characteristics Calculation}

Finally, the simulation calculates travel characteristics for the scheduled paratransit patrons by way of in-vehicle travel times, drive alone equivalent travel times, pickup time uncertainties, and arrival time delays. These travel characteristics are used in determining paratransit travel patron accessibility.

The first model estimates in-vehicle travel time, or the time, in minutes, it takes a patron to complete their paratransit trip. It comes from research conducted by Schofer et al. (13), and was estimated based on the 1995 NPTS. It is a nonlinear regression based on community size and travel distances.

The second linear regression model estimates drive alone equivalent travel time, or the time, in minutes, it would take a patron to make their paratransit trip if he/she was able to use a private vehicle and drove at an assumed average 30 mile per hour speed. The main predictors of drive alone equivalent travel time are the straight line distance and origin/destination census block group land areas. It was estimated using GIS straight census block group characteristics, straight line distance between census block group pairs, and shortest path travel times on the existing road network between census block group pairs. The full model specification is available from the authors, and is not being shown here to adhere to TRB word restrictions.

The third model estimates pickup time uncertainty, or the difference in time, in minutes, between the scheduled and actual pickup times, regardless of whether it is early or late. It was estimated from the BUS travel logs. Patrons traveling across the entire day (from morning to afternoon) tend to experience less time uncertainty than those traveling only in the morning or only in the afternoon. Those traveling for church, education, or shopping also tend to experience less time uncertainty. Travel during the slowest seasons, summer and winter, has lower time uncertainty. Interestingly, mobility-impaired patrons have the largest time uncertainty. The full model specification can be found in Table 4. 
The final exponential regression model estimates arrival time delay, or the number of minutes a patron will arrive late at their destination. It was estimated from the BUS travel logs. The results of this model, which can be found in Table 7, are generally similar to the previous one. Patrons traveling across the entire day (from morning to afternoon) tend to experience less delay than those traveling only in the morning; however, patrons traveling only in the afternoon are likely to experience more delay. Those traveling for church, medical, or shopping also tend to experience less delay. Travel during the slowest seasons, summer and winter, has lower delays. Interestingly, mobility-impaired patrons have the largest delay, perhaps due to the extra time required to help board and alight the vehicle.

\section{MEASURING PATRON ACCESSIBILITY}

Paratransit patron accessibility is calculated based on a number of relevant patron-level travel characteristics that describe how well each patron is being served by paratransit. These travel characteristics were identified from a survey of paratransit patrons in Tyler, Texas, and the weighting scheme was calculated by averaging respondents' rankings of each factor's importance to their trip satisfaction. They include the number of minutes late a patron arrives at their destination (weighted 50\%), the number of minutes difference between when a patron was scheduled to be picked up and when he/she actually was picked up (weighted 25\%), the difference in minutes between the time a patron spends in the paratransit vehicle and the equivalent time it would have taken if he/she was able to drive a personal vehicle (weighted $15 \%$ ), and the percent of the patrons from his/hers origin zone that were not able to be scheduled during this period (weighted 10\%). Patrons are most concerned with arriving at their destination on time and are less troubled by being picked up slightly early or late (as long as they get to their destination on time). Many paratransit patrons simply accept travel times since they do not have other travel options; however the third factor evokes service equity by comparing travel times for these patrons with their non-captive neighbors. Surprisingly, patrons were least concerned about not being served on their preferred day, perhaps due to the fact that they did not face this problem often.

The paratransit microsimulation patron accessibility analysis tool aggregates patron accessibility measures into zones, which allows for evaluation and comparison across the service region. In doing so, these patron accessibility values are scaled so that each zone is assigned a final relative patron accessibility index value between 0 and 1, with 1 representing high patron accessibility. Since these zone patron accessibility measures are relative to each other, there will always be one zone valued at 0 (the zone with the worst relative patron accessibility within the service region) as well as one zone valued at 1 (the zone with the best relative patron accessibility within the service region).

Because the microsimulation is done at the patron-level, meaning that an patron accessibility measure is calculated for each patron using the paratransit service, users have the option of calculating a general patron accessibility measure for each zone across all patrons, times of day, and trip purposes within each census block group or calculating a specific disaggregate patron accessibility measure for any combination of population groups, time of day, and trip purposes. For example, a user would be able to not only evaluate how accessible paratransit is generally for patrons from each zone but also how accessible paratransit is specifically for women going to work in the morning.

As a result, the paratransit microsimulation patron accessibility analysis tool allows

planners to evaluate current patron accessibility level for various combinations of population 
groups, service areas, times of day, and travel purposes. Much of the previous literature is focused on methods for benchmarking current practices, and the tool provides such detailed benchmarking measures of patron accessibility that can be compared over time. By using the analysis tool in this capacity, operators can study current service needs or evaluate patron accessibility trends over time. Furthermore, operators can measure patron accessibility spatially on a regional scale or focus on specific population groups/times/trip purposes to identify areas that need more reliable service or specific population groups that need to be targeted.

More importantly, however, is the ability to conduct "what if?" scenarios to evaluate changes in fleet characteristics (supply), population demographics (demand), and service areas (scope). The analysis tool allows an operator to calculate the impact of adding an additional vehicle or expanding the service region on patron paratransit patron accessibility. Similarly, operators can predict (and anticipate) future needs of their riders by using the tool to analyze changes in population demographics. Operators have the option of saving these scenarios for future comparison as well. These results have the potential to inform a range of public transportation planning, budgetary, and policy decisions. For example, purchasing an additional handicapped-accessible vehicle might be justified if mobility-impaired patron accessibility levels show a significant improvement with the addition of this vehicle. The analysis tool provides many such robust opportunities for the planner to see how any changes may impact transit operation. These "what if?" scenarios will become increasingly important as these small and medium sized communities continue to develop.

\section{CASE STUDY APPLICATION IN BROWNSVILLE, TEXAS}

The paratransit microsimulation patron accessibility analysis tool was applied to Brownsville, Texas, to assess paratransit patron accessibility and evaluate two of these 'what if?' scenarios (e.g., the impacts an additional fleet vehicle or an aging population have on patron accessibility). Brownsville is located within Cameron County at the southern tip of Texas and borders Mexico. As a result, Brownsville serves as a major gateway for people and goods between the two countries. It is not surprising that over $91 \%$ of the population is Hispanic and that over $87 \%$ speaks a language other than English at home. The city has an area of 83 square miles and is home to close to 140,000 residents (3). Brownsville is an ideal location to evaluate paratransit patron accessibility because the Brownsville Urban System (BUS) transit provider is widely considered to be one of the most advanced paratransit systems in Texas and the United States. BUS has an 11 vehicle paratransit fleet, 13 fixed transit routes, and 2 major terminals. BUS paratransit serves all those residents that live within 3/4-miles of the fixed-route transit network. Their paratransit service is supported by RouteMatch scheduling software and in-vehicle GPS transponders. In 2002, BUS served over 1.6 million passengers in its fixed route bus service and over 54 thousand passengers in its demand response transit services (3).

Figure 2a illustrates the current base paratransit patron accessibility for all patrons traveling in the morning for recreational trips, assuming all paratransit vehicles were running for the entire 14 hour service period. This type of travel is becoming increasingly important to consider in many small and medium-sized communities as paratransit operators are expanding their service to populations beyond ADA-mandated mobility-impaired patrons. It is important to recognize that the simulation included all patrons traveling for all purposes over the 24 hour period, but this analysis is focused in on just the 83 patrons who used the service during the morning for recreational activities. Each census block group has been assigned a relative patron accessibility index value that measures how easily patrons from that block group are getting to 
their desired recreational activity, relative to all other census block groups. These patron accessibility index values are ranked into three equal-sized groups (or quartiles) that represent the three levels of patron accessibility within the service region. The darkest census block groups are in the quartile identified as best serving paratransit patrons needs. As the census block groups coloring gets lighter, their relative level of patron accessibility diminishes. In this example, paratransit service provides the best patron accessibility for these patrons living in the areas along the main arterial corridors where street networks and land uses are more densely developed. Operators and planners can use maps such as this one to locate areas where paratransit service may need to be improved or further studied.

Figure $2 \mathrm{~b}$ illustrates the impact that one additional paratransit vehicle added to the fleet will have on patron accessibility. More specifically, this vehicle was defined as being able to transport five patrons per hour over the total service period. Patron accessibility has noticeably improved in the western half of the service region, where street networks and development are less dense. However, the number of patrons (76) has not changed dramatically, which is to be expected since none of the factors influencing demand have changed. This shift implies that the additional vehicle has provided additional flexibility in the system to improve travel times and reliability for patrons living further away from the recreational centers. It is important to note, however, that no matter what changes are made to the system, there will always be a scale to the patron accessibility indices (including upper and lower bounds of 1 and 0 , respectively), due to the fact that the indices are calculated relative to each other.

Alternatively, Figure 2c illustrates the impact that changes in the service region's characteristics will have on patron accessibility. In this example, planners have determined that over the next ten years that the number of patrons aged 50 and older will increase by $5 \%$ while the number of patrons aged 18 to 40 will decrease by the same amount. Again, relative patron accessibility improvements can be seen at the edges of the service region. However, the scale of the improvements cannot be compared across maps as the accessibility values are relative only within the same map. What is significant, nevertheless, is the dramatic increase and location of paratransit patrons. The population characteristics changed significantly enough to generate nearly double the number of patron (121), and these patrons are located further out from the urban center. Therefore, operators will need to prepare services to meet these changes in demand for the future.

\section{CONCLUSIONS AND FUTURE RESEARCH}

Over $21 \%$ of the United States' population currently resides in small and medium sized communities (5), and these numbers are projected to increase as such areas continue to develop as nationally critical economic centers (6). Unfortunately, paratransit transit systems within small and medium sized communities already face many challenges that restrict how well they can serve their community, including limited funding, understaffing, aging fleets, a lack of technical support, a lack of quantification of level of service standards, and reduced modeling/planning practices. As populations within such regions grow, these challenges will be amplified, resulting in potentially reduced mobility and stunted economic growth. Therefore, it is important that small and region communities take a proactive approach to transit planning. The paratransit microsimulation patron accessibility analysis tool presented in this paper can be applied to paratransit service regions of any size to assist transit operators in these efforts. In fact, the tool is specifically designed around practitioner needs and expertise. Operators can use the tool for two main planning applications: First, they can evaluate their current patron 
accessibility levels for various combinations of population groups, service areas, and travel purposes. Second, the patron accessibility analysis tool allows operators to undertake "what if?" scenarios to evaluate changes in fleet characteristics (supply), population demographics (demand), and service areas (scope).

Still, further research opportunities exist to better understand paratransit patron behavior. This is a unique form of transit that patrons need to plan ahead for, so their decision-making and travel behaviors are significantly different from those utilizing other modes; but they are still not well understood. Most important is the study of how paratransit patrons identify and select between different destinations to pursue activities. Similarly, researchers would benefit from studying the temporal aspects of paratransit travel as well as patrons' ability to trip chain using this service. Finally, patrons' perspectives naturally vary by individual, so it would be beneficial to further explore how perceptions of trip characteristics vary by demographics. Ultimately, results from these studies can help improve microsimulation techniques as well as general paratransit service operations.

\section{ACKNOWLEDGEMENTS}

The authors acknowledge the helpful comments of five anonymous reviewers on an earlier version of the paper. The authors would like to thank the Texas Department of Transportation for funding this research project. Thanks are also extended to Nazneen Ferdous and Prasad Vana for their assistance. Finally, the authors are grateful to Lisa Macias for her help in formatting this document. 


\section{REFERENCES}

1. Spielberg, F., and R. H. Pratt. TCRP Report 95: Traveler Response to Transportation System Changes, Chapter 6: Demand Responsive/ADA. Transportation Research Board, Washington, D.C., 2004.

2. Simon, R. M. Synthesis of Transit Practice 31: Paratransit Contracting and Service Delivery Methods. Transportation Research Board, Washington, D.C., 1998.

3. US Census Bureau. Fact Sheet: Brownsville, Texas. 2007. http://factfinder.census.gov/ Accessed on December 1, 2007.

4. Yim, Y. B., and J. Khattak. Personalized Demand Responsive Transit Systems. California PATH Research Report, August, 2000.

5. Northeast Midwest Institute. Rural Population as a Percent of State Total By State, 2000. 2002. http://nemw.org/poprural.htm Accessed on June 15, 2008.

6. Cambridge Systematics, Inc., Boston Logistics Group, Inc. and A. E. Pisarski. The Transportation Challenge: Moving the US Economy. National Chamber Foundation, Washington, D.C., 2008.

7. Kessler, D. S. TCRP Synthesis 57: Computer Aided Scheduling and Dispatch In Demand Responsive Transit Services. Transportation Research Board, Washington D.C., 2004.

8. KFH Group, Inc. and A-M-M-A. TCRP Report 70: Guidebook for Change and Innovation at Rural and Small Urban Transit Systems. Transportation Research Board, Washington, D.C., 2001.

9. KFH Group, Inc. TCRP Report 79: Effective Approaches to Meeting Rural Intercity Bus Transportation Needs. Transportation Research Board, Washington, D.C., 2002.

10. Conklin, J., C. Schweiger, B. Marks, W. Wiggins, and K. Timpone. Rural Transit ITS Best Practices. Federal Highway and US Department of Transportation, Washington, D.C., 2003.

11. Sandlin, A. B. and M. D. Anderson. Serviceability Index to Evaluate Rural DemandResponsive Transit System Operations. In Transportation Research Record: Journal of the Transportation Research Board, No. 1887, Transportation Research Board of the National Academies, Washington, D.C., 2004, 205-212.

12. Davenport, N. S., M. D. Anderson, and P. A. Farrington. Development and Application of a Vehicle Procurement Model for Rural Fleet Asset Management. In Transportation Research Record: Journal of the Transportation Research Board, No. 1927, Transportation Research Board of the National Academies, Washington, D.C., 2005, 123-127

13. Schofer, J. L., B. L. Nelson, R. Eash, M. Daskin, Y. Yang, H. Wan, J. Yan, and L. Medgyesy. TCRP Report 98: Resource Requirements for Demand Responsive Transportation Services. Transportation Research Board, Washington D.C., 2003.

14. Kittelson \& Associates, Inc., Urbitran, Inc., LKC Consulting Services, Inc., MORPACE International, Inc., Queensland University of Technology, Y. Nakanishi. TCRP Report 88: A Guidebook for Developing a Transit Performance-Measurement System. Transportation Research Board, Washington, D.C., 2003.

15. Bhat, C. R., S. Bricka, J. LaMondia, A. Kapur, J. Guo, and S. Sen. Metropolitan Area Transit Patron Accessibility Analysis Tool. Research Product Report 0-5178-P3, prepared for the Texas Department of Transportation, Center for Transportation Research, The University of Texas at Austin, 2006.

16. Potts, J. F., and M. A. Marshall. TCRP Synthesis 71: Parataransit Manager's Skills, Qualifications, and Needs. Transportation Research Board, Washington, D.C., 2007. 
17. Easter Seals Project ACTION. Innovative Practices in Paratransit Services. Washington, D.C., 2002.

18. KFH Group Inc. TCRP Report 124: Guidebook for Measuring, Assessing, and Improving Performance of Demand-Response Transportation. Transportation Research Board, Washington, D.C., 2008.

19. Victoria Transport Policy Institute. Rural Transportation Management: Improving Transportation Efficiency and Diversity in Rural Areas. 2008. http://www.vtpi.org/tdm/tdm87.htm Accessed on December 1, 2007.

20. US Department of Transportation. Rural Transport Toolbox. National Transportation Library, 2008. http://ntl.bts.gov/ruraltransport/toolbox/ Accessed on December 1, 2007.

21. Hanson, S. and G. Giuliano. The Geography of Urban Transportation. Third Edition. Guilford Press, New York, NY, 2004.

22. McIntyre, R. J., H. S. Mahmassani, C. M. Walton, and R. B. Machemehl. Rural Public Transportation Demand: Characteristics and Estimation Methodology. Technical Study Report 1080-1F. Texas Department of Transportation, 1986.

23. Koffman, D., D. Lewis, D. Chia, J. Burkhardt, and M. Bradley. TRCP Report 119: Improving ADA Complementary Paratransit Demand Estimation. Transportation Research Board, Washington D.C., 2007.

24. Painter, K., E. Jessup, M. Hill Gossard, and K. Casavant. Demand Forecasting for Rural Transit: Models Applied to Washington State. In Transportation Research Record: Journal of the Transportation Research Board, No. 2997, Transportation Research Board of the National Academies, Washington, D.C., 2007, 35-40.

25. Southworth, F., D. P. Vogt, and T. R. Curlee. Rural Transit Systems Benefits in Tennessee: Methodology and an Empirical Study. Environment and Planning A, Vol. 37, 2005, 861875.

26. Fu, L. A Simulation Model for Evaluating Advanced Dial-a-Ride Paratransit Systems. Transportation Research Part A, Vol. 36, 2002, 291-307.

27. Stommes, E. S. and D. M. Brown. Moving Rural Residents to Work: Lessons from Eight Job Access and Reverse Commute Projects. In Transportation Research Record: Journal of the Transportation Research Board, No. 1903, Transportation Research Board of the National Academies, Washington, D.C., 2005, 45-53.

28. Blumenberg, E. and K. Shiki. How Welfare Recipients Travel on Public Transit, and Their patron accessibility to Employment Outside Large Urban Centers. Transportation Quarterly, Vol. 57, No. 2, 2003, pp. 25-37.

29. Bhat, C. R., S. Srinivasan, J. Guo, and A. Sivakumar. Activity Based Travel Demand Analysis for Metropolitan Areas in Texas: A Micro-simulation Framework for Forecasting, Report 4080-4, prepared for the Texas Department of Transportation, Center for Transportation Research, The University of Texas at Austin, 2003. 


\section{LIST OF FIGURES}

FIGURE 124 hour paratransit patron travel simulation framework.

FIGURE 2a Current paratransit patron accessibility for morning recreation trips within Brownsville, TX.

FIGURE 2b Paratransit patron accessibility for morning recreation trips within Brownsville, $\mathrm{TX}$, with one additional vehicle in the fleet.

FIGURE 2c Paratransit patron accessibility for morning recreation trips within Brownsville, $\mathrm{TX}$, for project future populations.

\section{LIST OF TABLES}

TABLE 1 Total Patron Demand Generation Model Specification

TABLE 2 Destination Zone Assignement Model Specification

TABLE 3 Time of Day Allocation Model Specification

TABLE 4a Pickup Time Uncertainty Model Specification

TABLE 4b Arrival Time Delay Model Specification 


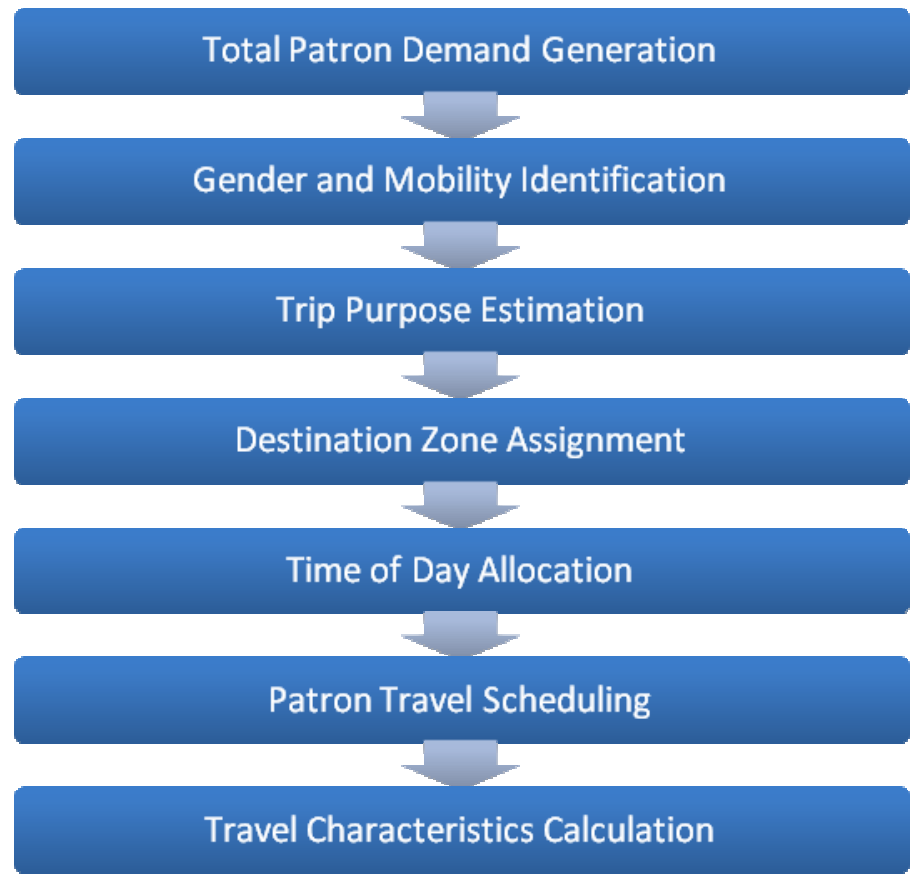

FIGURE 124 hour paratransit patron travel simulation framework. 


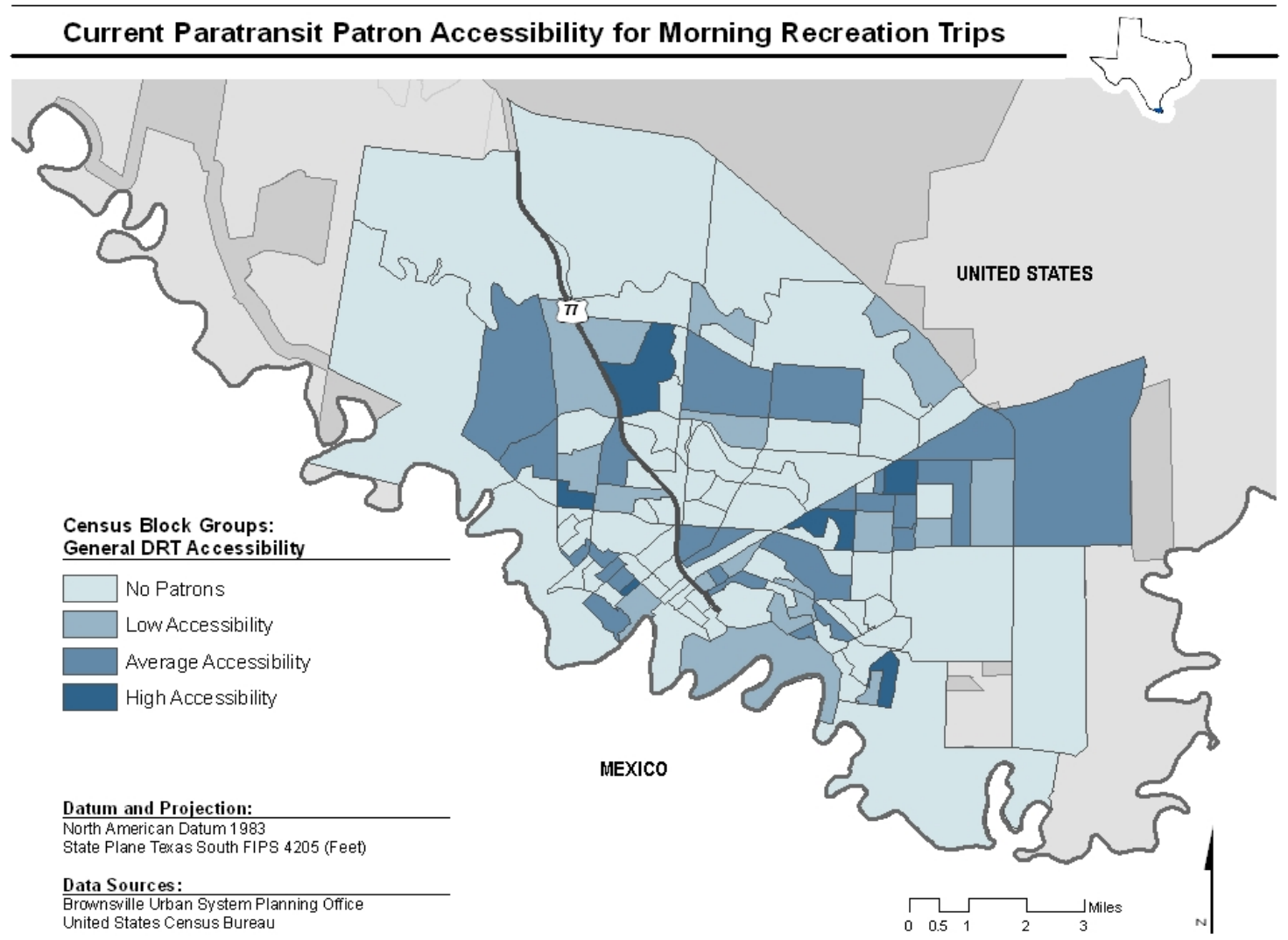

FIGURE 2a Current paratransit patron accessibility for morning recreation trips within Brownsville, TX. 


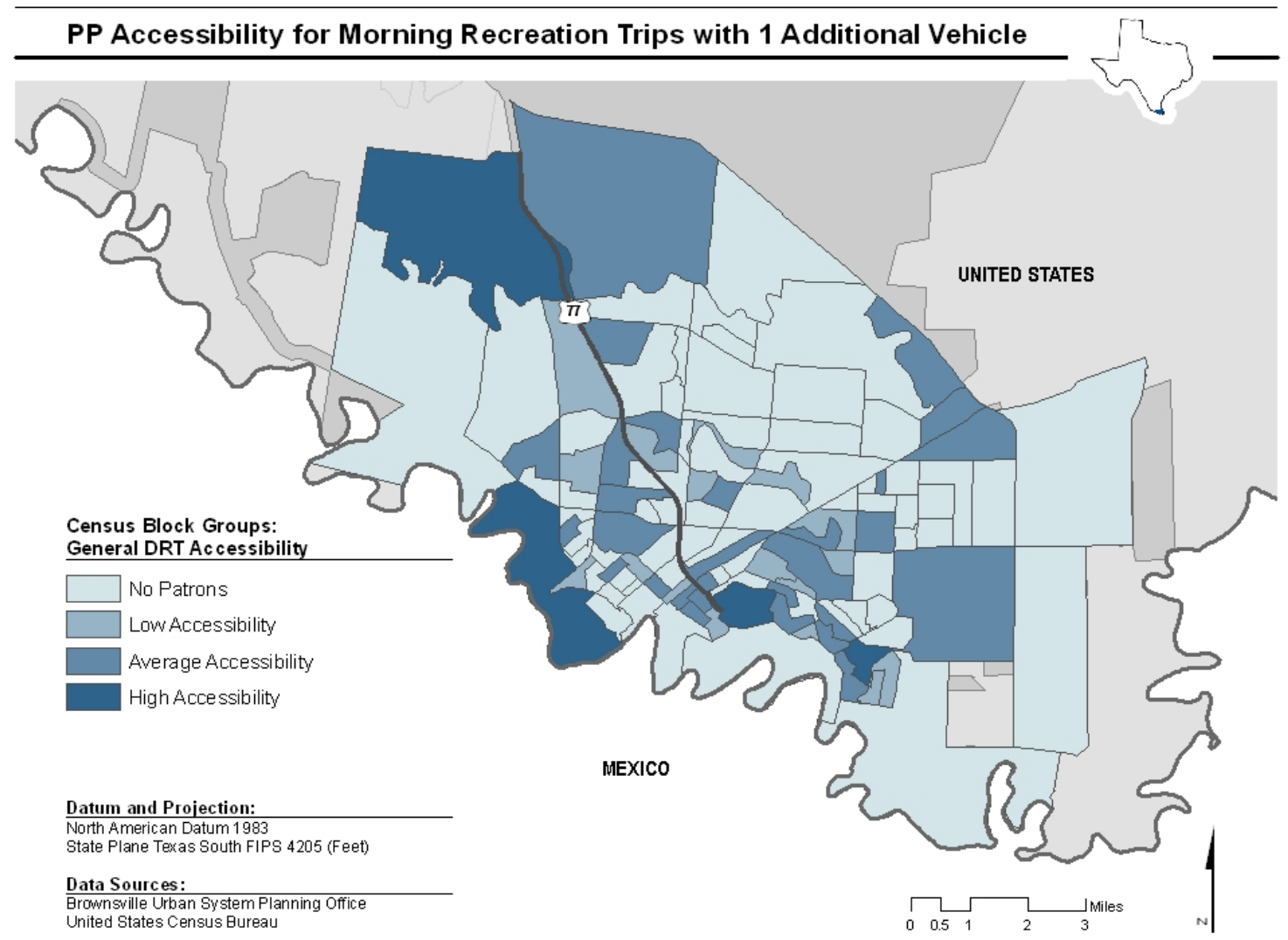

FIGURE 2b Paratransit patron accessibility for morning recreation trips within Brownsville, TX, with one additional vehicle in the fleet. 


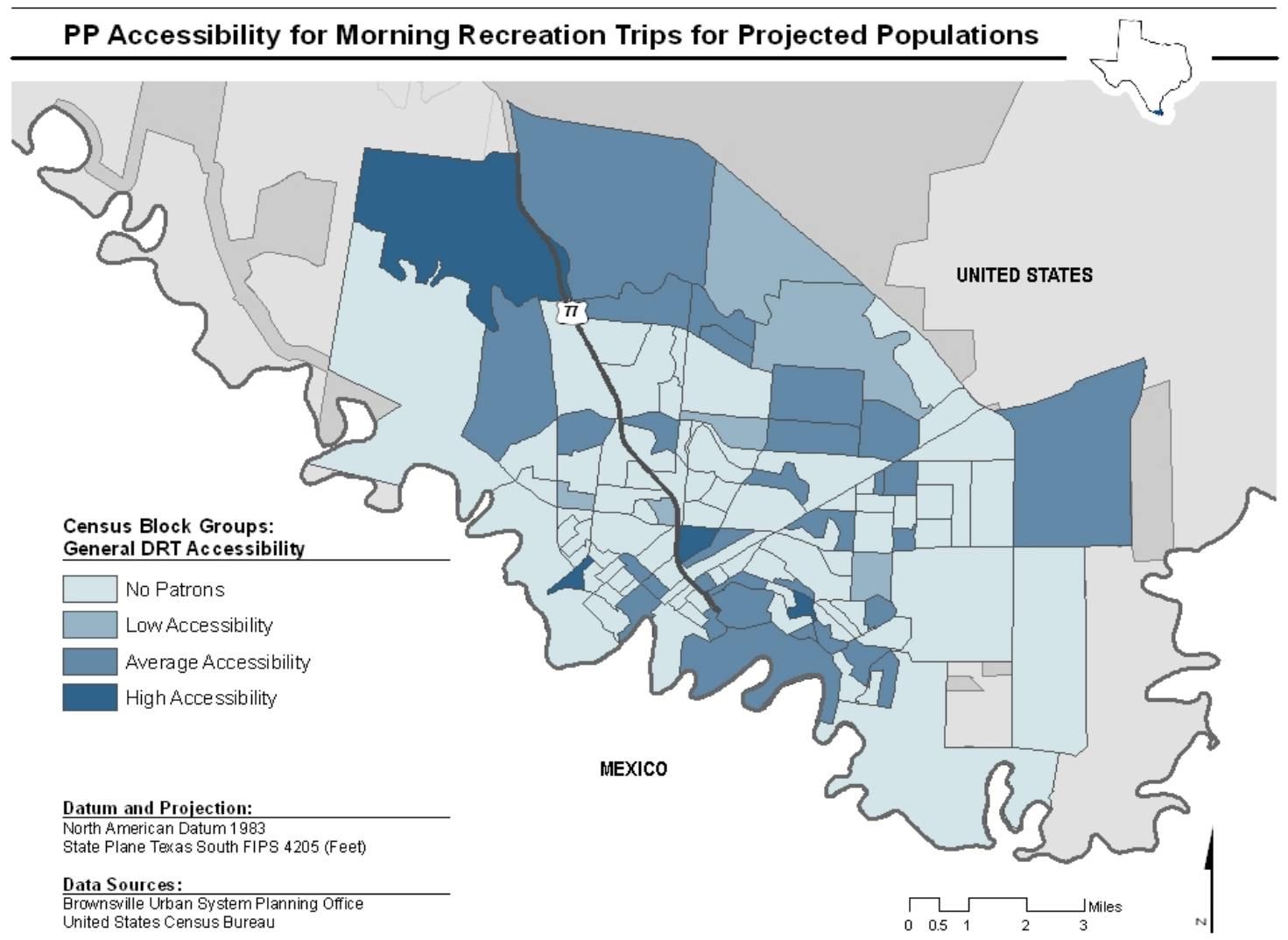

FIGURE 2c Paratransit patron accessibility for morning recreation trips within Brownsville, TX, for project future populations. 
TABLE 1 Total Patron Demand Generation Model Specification

\begin{tabular}{|c|c|c|}
\hline Parameters & Coefficient & t-stat \\
\hline Constant & -4.251 & -0.49 \\
\hline \multicolumn{3}{|l|}{ General Characteristics } \\
\hline Total Size of Census Block Group Population & 0.008 & 1.21 \\
\hline Median Age of Entire Census Block Group Population & -0.797 & -1.54 \\
\hline Median Age of Male Census Block Group Residents & 0.461 & 1.53 \\
\hline Median Age of Female Census Block Group Residents & 0.762 & 2.66 \\
\hline Average Household Size within Census Block Group & 2.315 & 1.84 \\
\hline Distance from Census Block Group Centroid to Nearest Transit Route, in miles & -0.702 & -1.20 \\
\hline \multicolumn{3}{|l|}{ Local Census Block Group Population Percentages } \\
\hline Percentage of Census Block Group Households that Rent & -7.649 & -2.39 \\
\hline Percentage of Census Block Group Households that are Married with Children & -0.247 & -2.63 \\
\hline Percentage of Census Block Group Population Aged 18-29 & 0.430 & 2.11 \\
\hline Percentage of Census Block Group Population Aged 50-64 & -0.477 & -1.91 \\
\hline Percentage of Census Block Group Population Aged 65 or Older & -0.772 & -3.30 \\
\hline \multicolumn{3}{|l|}{ Regional Population Percentages } \\
\hline Percentage of Service Region Renter Households within Census Block Group & 1.801 & 1.84 \\
\hline $\begin{array}{l}\text { Percentage of Service Region Married Households with Children within Census } \\
\text { Block Group }\end{array}$ & 4.407 & 1.14 \\
\hline $\begin{array}{l}\text { Percentage of Service Region Married Households without Children within } \\
\text { Census Block Group }\end{array}$ & -4.549 & -3.28 \\
\hline $\begin{array}{l}\text { Percentage of Service Region Population Aged 18-29 within Census Block } \\
\text { Group }\end{array}$ & -11.813 & -2.26 \\
\hline $\begin{array}{l}\text { Percentage of Service Region Population Aged 30-49 within Census Block } \\
\text { Group }\end{array}$ & -8.670 & -2.51 \\
\hline $\begin{array}{l}\text { Percentage of Service Region Population Aged 50-64 within Census Block } \\
\text { Group }\end{array}$ & 4.379 & 1.53 \\
\hline $\begin{array}{l}\text { Percentage of Service Region Population Aged } 65 \text { or Older within Census Block } \\
\text { Group }\end{array}$ & 5.074 & 2.03 \\
\hline $\begin{array}{l}\text { Sample Size: } 115 \text { Census Block Groups } \\
R^{2}: 0.494\end{array}$ & & \\
\hline
\end{tabular}


TABLE 2 Destination Zone Assignement Model Specification

\begin{tabular}{|c|c|c|}
\hline Parameters & Coefficient & t-stat \\
\hline \multicolumn{3}{|l|}{ Patron Characteristic Interactions (Base: Mobile Female) } \\
\hline Mobility-Impaired * Population Density of Census Block Group & -0.155 & -37.22 \\
\hline Mobility-Impaired * Distance from Census Block Group Centroid to Nearest Transit Route, in miles & -1.703 & -29.35 \\
\hline Male * Population Density of Census Block Group & -0.074 & -17.34 \\
\hline Male * Distance from Census Block Group Centroid to Nearest Transit Rout, in miles & -0.367 & -7.53 \\
\hline \multicolumn{3}{|l|}{ Trip Purpose Interactions (Base: Travel to Work) } \\
\hline Travel to Church/Meeting * Percent of Census Block Group Area Zoned for Apartments & 0.726 & 2.24 \\
\hline Travel to Church/Meeting * Percent of Census Block Group Area Zoned for Commercial/Public Buildings and Space & 0.200 & 1.63 \\
\hline Travel to Church/Meeting * Percent of Census Block Group Area Zoned for Retail Business & 0.922 & 8.43 \\
\hline Travel to Education/School * Percent of Census Block Group Area Zoned for Apartments & -6.681 & 20.36 \\
\hline Travel to Education/School * Percent of Census Block Group Area Zoned for Commercial/Public Buildings and Space & 1.021 & 10.98 \\
\hline Travel to Education/School * Percent of Census Block Group Area Zoned for Manufacturing/Industrial & -2.083 & -7.11 \\
\hline Travel to Education/School * Percent of Census Block Group Area Zoned for Retail Business & 1.457 & 16.44 \\
\hline Travel to Recreation * Percent of Census Block Group Area Zoned for Apartments & 1.975 & 10.85 \\
\hline Travel to Recreation * Percent of Census Block Group Area Zoned for Commercial/Public Buildings and Space & 1.449 & 21.88 \\
\hline Travel to Recreation * Percent of Census Block Group Area Zoned for Retail Business & 1.089 & 15.06 \\
\hline Travel to Medical/Therapy * Percent of Census Block Group Area Zoned for Apartments & 1.797 & 16.60 \\
\hline Travel to Medical/Therapy * Percent of Census Block Group Area Zoned for Commercial/Public Buildings and Space & 0.206 & 4.54 \\
\hline Travel to Medical/Therapy * Percent of Census Block Group Area Zoned for Manufacturing/Industrial & -0.365 & -4.46 \\
\hline Travel to Shopping * Percent of Census Block Group Area Zoned for Retail Business & 0.604 & 4.02 \\
\hline $\begin{array}{l}\text { Sample Size: } 17770 \text { Patrons } \\
\text { Log-Likelihood at Convergence: -81848.2646 }\end{array}$ & & \\
\hline
\end{tabular}


TABLE 3 Time of Day Allocation Model Specification

\begin{tabular}{|c|c|c|c|c|}
\hline \multirow[b]{3}{*}{ Parameters } & \multicolumn{4}{|c|}{$\begin{array}{l}\text { Travel Time of Day } \\
\text { (Base: Travel Out in the AM and Return in the AM) }\end{array}$} \\
\hline & \multicolumn{2}{|c|}{$\begin{array}{l}\text { Travel Out in the AM } \\
\text { and Return in the PM }\end{array}$} & \multicolumn{2}{|c|}{$\begin{array}{l}\text { Travel Out in the PM } \\
\text { and Return in the PM }\end{array}$} \\
\hline & Coeff. & t-stat & Coeff. & t-stat \\
\hline $\begin{array}{l}\text { Patron Characteristics (Base: Mobile) } \\
\quad \text { Mobility-Impaired }\end{array}$ & -0.362 & -5.72 & -1.012 & -12.25 \\
\hline $\begin{array}{l}\text { Trip Characteristics } \\
\text { Distance Between Origin and Destination Census } \\
\text { Block Group Centroids, in miles }\end{array}$ & -0.024 & -6.13 & - & - \\
\hline $\begin{array}{l}\text { Trip Purpose (Base: Travel to Work) } \\
\text { Travel to Church/Meeting } \\
\text { Travel to Education/School } \\
\text { Travel to Recreation } \\
\text { Travel to Medical/Therapy } \\
\text { Travel to Shopping }\end{array}$ & $\begin{array}{r}0.298 \\
-0.911 \\
1.346 \\
-1.318 \\
-0.785\end{array}$ & $\begin{array}{r}2.05 \\
-8.23 \\
10.96 \\
-20.51 \\
-5.65\end{array}$ & $\begin{array}{r}1.682 \\
-1.688 \\
0.547 \\
-0.182 \\
0.661\end{array}$ & $\begin{array}{r}10.74 \\
-10.56 \\
3.58 \\
-2.13 \\
4.55\end{array}$ \\
\hline $\begin{array}{l}\text { Sample Size: } 6686 \text { Patrons } \\
\text { Log-Likelihood at Convergence: }-5861.0680\end{array}$ & & & & \\
\hline
\end{tabular}




\section{TABLE 4a Pickup Time Uncertainty Model Specification}

\begin{tabular}{|l|c|c|}
\hline \multicolumn{1}{|c|}{ Parameters } & Coefficient & t-stat \\
\hline Constant & 2.368 & 61.00 \\
\hline $\begin{array}{l}\text { Travel Time of Day (Bases: Travel Only in the AM or Only in the PM) } \\
\text { Travel Out in the AM and Return in the PM }\end{array}$ & -0.286 & -8.77 \\
\hline $\begin{array}{l}\text { Trip Purpose (Base: Travel to Work) } \\
\text { Travel to Church/Meeting } \\
\text { Travel to Education/School } \\
\text { Travel to Shopping }\end{array}$ & -0.310 & -3.84 \\
\hline $\begin{array}{l}\text { Travel Season (Bases: Travel in Spring or Fall) } \\
\text { Travel in the Summer }\end{array}$ & -0.194 & -2.66 \\
$\quad$ Travel in the Winter & -0.131 & -1.45 \\
\hline $\begin{array}{l}\text { Patron Characteristics } \\
\text { Mobility-Impaired (Base: Mobile) }\end{array}$ & -0.154 & -3.79 \\
\hline $\begin{array}{l}\text { Sample Size: 6686 Patrons } \\
R^{2} \text { 0.152 }\end{array}$ & -0.072 & -1.75 \\
\hline
\end{tabular}


TABLE 4b Arrival Time Delay Model Specification

\begin{tabular}{|c|c|c|}
\hline Parameters & Coefficient & t-stat \\
\hline Constant & 1.575 & 25.70 \\
\hline $\begin{array}{l}\text { Travel Time of Day (Base: Travel Only in the AM or Only in the PM) } \\
\text { Travel Out in the AM and Return in the PM } \\
\text { Travel Out in the PM and Return in the PM }\end{array}$ & $\begin{array}{r}-0.247 \\
0.122\end{array}$ & $\begin{array}{r}-5.64 \\
2.21\end{array}$ \\
\hline $\begin{array}{l}\text { Trip Purpose (Base: Travel to Work) } \\
\text { Travel to Church/Meeting } \\
\text { Travel to Education/School } \\
\text { Travel to Recreation } \\
\text { Travel to Medical/Therapy } \\
\text { Travel to Shopping }\end{array}$ & $\begin{array}{l}-0.324 \\
-0.199 \\
-0.194\end{array}$ & $\begin{array}{l}-3.50 \\
-4.88 \\
-1.89\end{array}$ \\
\hline $\begin{array}{l}\text { Travel Season (Base: Travel in Spring or Fall) } \\
\text { Travel in the Summer } \\
\text { Travel in the Winter }\end{array}$ & $\begin{array}{l}-0.199 \\
-0.267\end{array}$ & $\begin{array}{l}-4.50 \\
-5.95\end{array}$ \\
\hline $\begin{array}{l}\text { Patron Characteristics (Base: Mobile Female) } \\
\text { Mobility-Impaired } \\
\text { Male }\end{array}$ & $\begin{array}{r}0.165 \\
-0.049\end{array}$ & $\begin{array}{r}4.46 \\
-1.39\end{array}$ \\
\hline $\begin{array}{l}\text { Sample Size: } 6686 \text { Patrons } \\
R^{2}: 0.023\end{array}$ & & \\
\hline
\end{tabular}

